\title{
Schweizer Empfehlungen für das Management des Herpes genitalis und der Herpes-simplex- Virus-Infektion des Neugeborenen
}

\author{
Swiss Herpes Management Forum*
}

Herpes genitalis wird als ein medizinisches Problem von zunehmender Bedeutung erkannt. Diagnose und Management sind komplex. Die vorliegenden Empfehlungen wurden von einer interdisziplinären Expertengruppe erarbeitet und von all jenen Schweizerischen Fachgesellschaften gutgeheissen, die solche Patienten betreuen (Appendix). Das Ziel ist, die Betreuung der betroffenen Patienten zu verbessern, die horizontale und vertikale Übertragung zu reduzieren sowie die psychosoziale Last zu verringern.

Version française: $w w w . b u l l m e d . c h / p d f / r e c h e r p e s . p d f$

Versione italiana: $w w w . s a e z . c h / p d f / r a c h e r p e s . p d f$

\footnotetext{
* Mitglieder des Swiss Herpes Management Forums:

S. Büchner, Dermatologie, Universitätsspital Basel; P. Erni, GlaxoSmithKline, Münchenbuchsee; J. Garweg, Augenklinik, Inselspital Bern; S. Gerber, Gynäkologie und Geburtshilfe, CHUV Lausanne; H. H. Hirsch, Medizinische Poliklinik, Universitätsspital Basel; W. Kempf, Dermatologie, Universitätsspital Zürich; C. Kind, Ostschweizerisches Kinderspital, St. Gallen; U. Lauper, Gynäkologie und Geburtshilfe, Universitätsspital Zürich; S. Lautenschlager, Dermatologisches Ambulatorium Triemli, Zürich; P. Meylan, Institut für Mikrobiologie, Abteilung für infektiöse Krankheiten, CHUV Lausanne; D. Nadal, Infektiologie und Spitalhygiene, Universitätskinderklinik Zürich; P. Reusser, Innere Medizin, Hôpital du Jura Porrentruy; W. Wunderli, HUG Genève.
}

Unterstützt von

GlaxoSmithKline AG.

Korrespondenz:

Prof. Dr. S. Büchner

Universitätsspital Basel

Dermatologische Poliklinik

Petersgraben 4

CH-4031 Basel

Tel. 0612654087

Fax 0612655742

E-Mail: sbuechner@uhbs.ch

\section{Einleitung}

Dieses Dokument richtet sich an praktizierende Ärzte. Die Empfehlungen wurden auf der Basis von kürzlich in Europa und den Vereinigten Staaten veröffentlichten Direktiven [1, 2] erarbeitet, berücksichtigen aber aktuelle Ergebnisse zur Virologie, Klinik und Behandlung der Herpesinfektionen sowie Besonderheiten der Schweizer Pharmakopöe. Die Qualität der Empfehlungen wurde entsprechend der vorhandenen Evidenz gewichtet und kodifiziert (Tab. 1).

\section{Tabelle 1}

Evidenzen und Empfehlungen.
Herpes genitalis ist eine durch muko-kutanen Kontakt erworbene Viruserkrankung. Die Primärinfektion manifestiert sich klinisch unterschiedlich und bleibt mehrheitlich asymptomatisch [3]. Ist sie jedoch symptomatisch, so verläuft die Primärinfektion mit lokalen und oft auch systemischen Zeichen [4]. Beide Herpessimplex-Virus-Typen (HSV-1 und HSV-2) können für die Primärinfektion verantwortlich sein, wobei die Proportion von HSV-1 in Europa zunimmt [5, 6]. Diese Viren etablieren eine latente Infektion in den sensorischen sakralen Ganglien, wo es zu ihrer Reaktivierung mit oder ohne klinisch-symptomatischen Rezidiven kommt. Die Rezidivhäufigkeit variiert von einem Patienten zum anderen und nimmt individuell mit den Jahren nach der Primärinfektion ab [7]. Im Genitalbereich reaktiviert HSV-2 häufiger als HSV1. Die Prävalenz von HSV-1 und HSV-2 kann durch typspezifische IgG bestimmt werden. Etwa $70 \%$ der erwachsenen Schweizer sind mit HSV-1 infiziert, etwa $20 \%$ mit HSV-2 [8]. Es wird geschätzt, dass von fünfundzwanzig Schweizern einer an einem diagnostizierten, rezidivierenden Herpes genitalis leidet, drei einen nicht diagnostizierten Herpes genitalis haben und bei einem eine asymptomatische Infektion vorliegt.

\begin{tabular}{ll}
\hline Evidenz & Studientyp \\
\hline II & Kontrollierte und randomisierte Studien (oder systematische Durchsicht solcher Studien) \\
\hline III & Kontrollierte, jedoch nicht randomisierte Studie \\
\hline IV & Prospektive Kohortenstudie \\
\hline V & Retrospektive Kohortenstudie oder Fallkontrollstudie \\
\hline Empfehlung & Fallreihenstudie, Expertenmeinung \\
\hline A & Evidenz \\
\hline B & Solide Beweise zur Untermauerung der Empfehlung (im allgemeinen Evidenz I) \\
\hline C & Akzeptable Beweise zur Untermauerung der Empfehlung (im allgemeinen Evidenz II oder III) \\
\hline D & Ungenügende Beweise; Empfehlung basiert auf anderen Gründen \\
\hline E & Akzeptable Beweise gegen die Empfehlung (im allgemeinen Evidenz II oder III) \\
\hline
\end{tabular}




\section{2. Übertragung}

Ein Übertragungsrisiko existiert trotz fehlender Symptome. Die meisten horizontalen (zwischen Partnern) oder vertikalen (Mutter-Kind-) Übertragungen ereignen sich während einer asymptomatisch verlaufenden Infektion [9, 10] (III). Das Risiko einer Übertragung vom Mann auf die Frau ist ungefähr fünfmal höher als umgekehrt (III). Latexpräservative reduzieren das Infektionsrisiko (C). Eine signifikante Reduktion des Infektionsrisikos mit HSV-2 von Mann zu Frau konnte in einer Studie [11] (I) bei Verwendung von Latexpräservativen in mindestens $25 \%$ der Sexualkontakte gezeigt werden. Zur Risikoreduktion von Frau zu Mann mittels Latexpräservativen gibt es bisher keine Daten. Die suppressive Behandlung mit Valaciclovir verringert die HSV2-Übertragung bei Partnern mit diskordantem serologischem Status um 50\% [12] (I). Die steigende Verbreitung orogenitaler Sexualpraktiken erklärt die Zunahme der Häufigkeit von HSV-1 bei der initialen Episode des Herpes genitalis [13] (III). Eine vorgängige Infektion mit HSV-1 scheint nicht vor einer Infektion mit HSV-2 zu schützen, vermindert jedoch die Wahrscheinlichkeit einer symptomatischen Herpeserkrankung [3].

Herpes genitalis begünstigt die Übertragungswahrscheinlichkeit des HIV bei HSV-2seropositiven Personen [14] (III). Deshalb trägt jede Massnahme zur Vorbeugung der Übertragung von HSV-2 indirekt auch zur Vorbeugung einer Übertragung von HIV bei (B).

\section{Diagnose}

\subsection{Anamnese und Präsentationen}

\subsubsection{Primärinfektion}

Bei mehr als der Hälfte der Patienten wird die Primärinfektion nicht bemerkt [3]. Wenn Symptome präsent sind, erscheint ein primärer Herpes genitalis drei Tage bis zwei Wochen nach Kontakt mit einem infizierten Sexualpartner und manifestiert sich als gruppierte, schmerzhafte Bläschen, die nach mehreren Tagen in Ulzerationen übergehen. Die Primärinfektion verläuft typischerweise mit ausgedehnten multiplen Ulzerationen und schmerzhaften Lymphknotenschwellungen. Mehr als die Hälfte der Patienten leidet an Allgemeinsymptomen. Bis zu 20\% weisen eine Dysurie auf, selten kann es zu akutem Harnverhalten führen. Ein Drittel der Frauen weist komplizierend eine aseptische Meningitis auf, was bei Männern seltener vorkommt [15].
Ein vollständiges Abheilen der Läsionen wird nach drei Wochen erreicht. Beide HSV-Typen provozieren ein identisches klinisches Bild.

Nichtprimärepisoden oder initiale Infektionen bei Vorhandensein von HSV-Antikörpern resultieren in milderen Episoden mit selteneren Allgemeinsymptomen. In der Praxis ist eine klinische Unterscheidung zwischen Primärinfektion und Initialinfektion praktisch unmöglich.

\subsubsection{Rezidivierender Herpes genitalis}

Im Genitaltrakt kann das HSV asymptomatisch oder symptomatisch reaktivieren. Klassische Symptome sind gruppierte Bläschen, die in Ulzeration und Krusten übergehen. Läsionen sind typischerweise seltener und kleiner als bei der Primärinfektion. Allgemeinsymptome sind selten [16]. Bis zu 89\% der Patienten mit einer symptomatischen ersten Episode aufgrund von HSV-2 leiden an symptomatischen Rezidiven von unterschiedlicher Häufigkeit und Schwere [17] (III). Die Rezidivhäufigkeit in der Genitalregion ist für HSV-2 viel grösser als für HSV-1 [18] (III). Rezidiven gehen oft Prodromi wie Brennen oder Jukken an der Ausbruchstelle oder neuralgiformer Schmerz voraus.

Bei vielen Patienten sind Läsionen und Lokalisation jedoch weniger typisch und werden ohne gezielte Information und spezifische Diagnostik nicht als Herpes genitalis erkannt [16, $19,20]$ (III). Informiert man detailliert Patienten, die nach eigenen Angaben keinen Herpes genitalis, jedoch eine serologisch nachgewiesene HSV2-Infektion haben, wird sich die Hälfte anschliessend als symptomatisch beschreiben [20] (III).

Solche atypischen Lokalisationen (im Gesäss-, Anal- oder Oberschenkelbereich) sind relativ häufig (bei Frauen bis zu 61\%) und manchmal von neuralgiformen Schmerzen begleitet [21] (IV). Eine Labordiagnostik zur Bestätigung ist folglich gerechtfertigt (C).

Mit steigender Anzahl von Patienten mit eingeschränkter Immunität, z. B. nach Transplantation oder HIV-Infektion, nehmen atypische Präsentationen mit chronischen und extensiveren Läsionen zu.

Verschiedene Klassifizierungen von Herpes genitalis werden in Tabelle 2 aufgeführt.

\subsection{Nachweis von Infektionen mit Herpes-simplex-Viren}

Herpesvirusinfektionen können auf zwei verschiedene Arten identifiziert werden: durch den Nachweis des Virus oder eines seiner Bestandteile in einer Probe (Tab. 3) oder durch die Bestimmung spezifischer Antikörper im Blut gegen diese Viren [22-24]. 


\subsubsection{Virusnachweis}

Es gibt eine Vielzahl von Nachweismethoden, die aber im Prinzip auf die drei Grundmethoden zurückgeführt werden können:

- Virusnachweis mittels Zellkultur;

- Virusantigennachweis mittels spezifischer Antikörper (Enzymimmunoassay oder Immunfluoreszenz);

- Nachweis von Sequenzen des Virusgenoms nach enzymatischer Amplifikation (Polymerasen Kettenreaktion $=$ PCR).

Deren Eigenschaften und Verwendung sind in Tabelle 3 dargestellt.

\subsubsection{Antikörpernachweis}

Mit dem Nachweis von typspezifischen IgG gegen das Glykoprotein G von HSV-1 (gG-1) oder das Glykoprotein G von HSV-2 (gG-2) kann die Diagnose einer Infektion mit HSV-1 oder HSV-2 in Abwesenheit von Läsionen beim Patienten oder seinem Sexualpartner gestellt werden. In kommerziell erhältlichen Produkten, welche typspezifische IgG unterscheiden können, werden vorwiegend synthetische Glykoproteine verwendet (siehe BAG-Bulletin und Swissmedic: www.bag.admin.ch/md/ivd/list_h.pdf).

Trotz der Bedeutung des Nachweises von typspezifischen IgG in Partnerbeziehungen und
STD-Kliniken gibt es bestimmte Grenzen der typspezifischen Serologie. Ein negatives serologisches Resultat für einen Typ (HSV-1 oder HSV-2) erlaubt nicht automatisch, auf eine Primoinfektion zu schliessen:

- Nach HSV-Primärinfektion kann es Wochen bis Monate dauern, bis die typspezifische Immunantwort voll ausgebildet ist. Für IgG gegen gG-2 dauert dies im Durchschnitt etwa 8 Wochen, bei gG-1 dauert es noch länger. Eine Behandlung mit antiviralen Medikamenten kann zusätzlich die Immunantwort (Serokonversion) verzögern.

- IgG gegen gG-1 und gG-2 können allmählich wieder verschwinden, wenn der latent infizierte Patient während längerer Zeit nicht exponiert wurde oder keine Reaktivierung durchgemacht hat [25].

- In sehr seltenen Fällen ist die humorale Immunantwort nicht gegen das in den Tests verwendete Glykoprotein (gG-2) gerichtet.

\section{Therapie des Herpes genitalis}

Antivirale Medikamente sind eine zentrale Komponente der Therapie des Herpes genitalis. Manifestation (erstmalig, rezidivierend), Patientencharakteristik (immunkompetent, immun-

Tabelle 2

Verschiedene Klassifizierungen von Herpes-genitalis-Infektionen.

\begin{tabular}{|c|c|c|c|}
\hline Klinik & $\begin{array}{l}\text { Infizierender } \\
\text { HSV-Typ }\end{array}$ & HSV-Antikörper & $\begin{array}{l}\text { Klassifizierung } \\
\text { der Infektion }\end{array}$ \\
\hline \multirow{5}{*}{$\begin{array}{l}\text { Als Herpes genitalis erkannte Symptome: } \\
\text { erste Episode }\end{array}$} & HSV-2 & Keine & Primärer HSV-2 \\
\hline & HSV-1 & Keine & Primärer HSV-1 \\
\hline & HSV-2 & HSV-2 & Nicht primärer HSV-2 \\
\hline & HSV-1 & HSV-1 & Nicht primärer HSV-1 \\
\hline & HSV-2 & HSV-1 & Initialer HSV-2 \\
\hline \multirow[t]{2}{*}{$\begin{array}{l}\text { Als Herpes genitalis erkannte Symptome: } \\
\text { rezidivierender Herpes }\end{array}$} & HSV-2 & $\begin{array}{l}\text { HSV-2 mit oder ohne } \\
\text { HSV-1 }\end{array}$ & Rezidivierender HSV-2 \\
\hline & HSV-1 & $\begin{array}{l}\text { HSV-1 mit oder ohne } \\
\text { HSV-2 }\end{array}$ & Rezidivierender HSV-1 \\
\hline \multirow[t]{2}{*}{ Absenz einer Herpes-genitalis-Episode } & HSV-2 & $\begin{array}{l}\text { HSV-2 mit oder ohne } \\
\text { HSV-1 (theoretisch } \\
\text { auch keine) }\end{array}$ & $\begin{array}{l}\text { Asymptomatische } \\
\text { Ausscheidung von } \\
\text { HSV-2 }\end{array}$ \\
\hline & HSV-1 & $\begin{array}{l}\text { HSV-1 mit oder ohne } \\
\text { HSV-2 (theoretisch } \\
\text { auch keine) }\end{array}$ & $\begin{array}{l}\text { Asymptomatische } \\
\text { Ausscheidung von } \\
\text { HSV-1 }\end{array}$ \\
\hline \multirow[t]{2}{*}{ Symptome nicht als Herpes genitalis erkannt } & HSV-2 & $\begin{array}{l}\text { HSV-2 mit oder ohne } \\
\text { HSV-1 (theoretisch } \\
\text { auch keine) }\end{array}$ & $\begin{array}{l}\text { Unerkannter } \\
\text { symptomatischer } \\
\text { genitaler HSV-2 }\end{array}$ \\
\hline & HSV-1 & $\begin{array}{l}\text { HSV-1 mit oder ohne } \\
\text { HSV-2 (theoretisch } \\
\text { auch keine) }\end{array}$ & $\begin{array}{l}\text { Unerkannter } \\
\text { symptomatischer } \\
\text { genitaler HSV-1 }\end{array}$ \\
\hline
\end{tabular}




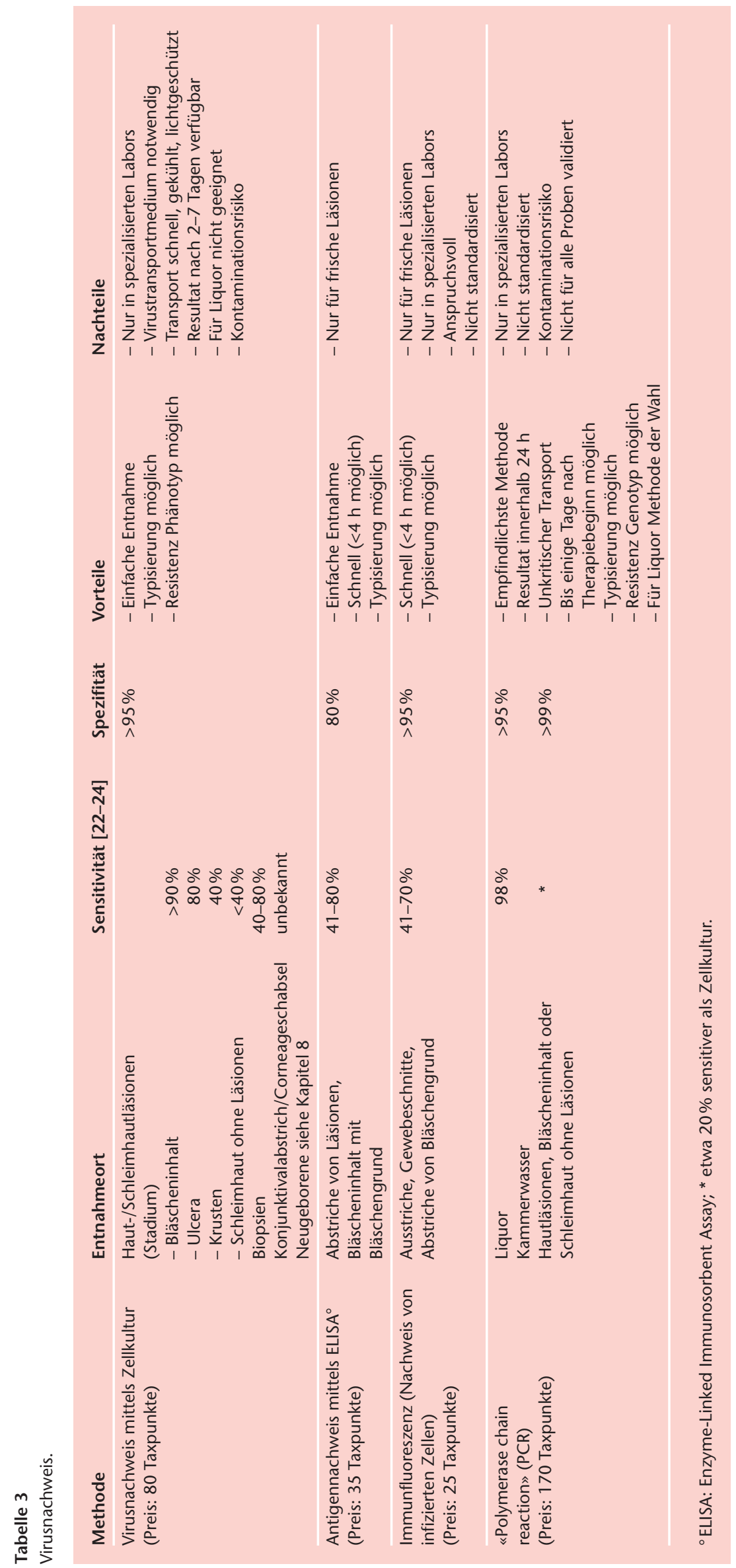


supprimiert, schwanger, neonatal) und Zielsetzung der Behandlung (therapeutisch-episodisch, prophylaktisch-suppressiv) müssen berücksichtigt werden.

Systemisch angewandte antivirale Medikamente vermindern die Symptome des Herpes genitalis bei der Behandlung der Erstepisode und auch - zu einem geringeren Grad - der Rezidive. Als suppressive Therapie eingesetzt senken diese Medikamente die Rezidivhäufigkeit signifikant. Sie eradizieren jedoch die latente Infektion nicht. Die Häufigkeit und die Schwere der Rezidive werden nach Absetzen der suppressiven Behandlung nicht modifiziert. Randomisierte Studien weisen darauf hin, dass drei Arzneimittel eine klinische Wirkung auf den Herpes genitalis haben: Aciclovir, Valaciclovir und Famciclovir. Valaciclovir, der L-Valinester von Aciclovir, weist eine deutlich bessere Bioverfügbarkeit auf als Aciclovir. Famciclovir, ein Prodrugester von Penciclovir, zeichnet sich ebenfalls durch eine hohe Bioverfügbarkeit aus.

Eine topische Behandlung mit antiviralen Medikamenten ist nicht von nachweisbarem klinischem Nutzen [26] (I) und wird generell nicht empfohlen (E).

\subsection{Therapie der Erstepisode}

Die oft schweren Symptome rechtfertigen eine antivirale Behandlung, die so früh als möglich und ohne Abwarten der Laborergebnisse eingeleitet werden soll (A). Bei Behandlungbeginn innerhalb der ersten 5 Tage nach Beginn der Symptome klingen diese fast eine Woche früher ab [27-30]. Die empfohlenen Therapien sind in Tabelle 4 aufgeführt. Die in den USA gebräuchliche Dosierung von Valaciclovir von $2 \times 1000 \mathrm{mg}$ täglich beruht auf den Studienergebnissen von Fife et al. [30] (A). Da mit $2 \times 500 \mathrm{mg}$ Valaciclovir jedoch eine höhere Plasmakonzentration als mit $5 \times 200 \mathrm{mg}$ Aciclovir erreicht wird [31], wird in Europa die Dosierung von $2 \times 500 \mathrm{mg}$ Valaciclovir täglich bevorzugt (A). Die klinische Wirksamkeit von $2 \times 500 \mathrm{mg}$ konnte in einer kontrollierten Studie bestätigt werden [32] (I). Bei protrahiertem klinischem Bild mit persistierenden Läsionen, insbesondere bei immungeschwächten Patienten, beträgt die Behandlungsdauer mindestens 14 Tage. Aciclovir, Valaciclovir und Famciclovir scheinen von vergleichbarer Wirksamkeit zu sein; die Wahl beruht auf den Kriterien der Verträglichkeit, Therapietreue und Kosten. Die Behandlung der Erstepisode beeinflusst die Rezidivrate nicht [33-35] (I).

Folgende Komplikationen erfordern eine Hospitalisation: Sphinkterstörungen mit Harnverhalten, Meningismus, schwere Allgemein- symptome, Unmöglichkeit, Medikamente oral einzunehmen. Bei Harnverhalt kann für ein paar Tage ein Dauerkatheter gelegt werden, wobei eine suprapubische Ableitung bevorzugt wird (C).

Analgetika werden empfohlen. Lokalanästhetika sind aufgrund der Sensibilisierungsgefahr zurückhaltend einzusetzen. Empfehlenswert ist eine antiseptische und antiphlogistische Behandlung mit iodhaltigen oder adstringierenden Lösungen (Gerbstoffe) mittels Sitzbädern oder Kompressen (C).

\subsection{Therapie der Rezidive}

Je nach Ausprägung der Rezidive erfolgt eine Therapie, episodisch oder suppressiv, oder eine alleinige unspezifische Lokaltherapie. Die Wahl muss nach Besprechung und im Einvernehmen jeweils individuell mit dem Patienten getroffen werden. Eine episodische Behandlung während 2-5 Tagen (Tab. 4), die innerhalb von 24 Stunden nach Einsetzen der Symptome durch den Patienten selbst eingeleitet wird, kann die Dauer einer Episode um 1 bis 2 Tage verkürzen [29, 36-42]. Eine suppressive Behandlung kann rund $80 \%$ der Rezidive unterdrücken und hat eine viel grössere Wirkung auf die mit dem Herpes genitalis verbundene Morbidität [43] (I).

\subsubsection{Episodische Behandlung}

Angesichts der Notwendigkeit einer raschen Einnahme der Medikamente bei Auftreten der Symptome muss dem Patienten entweder eine antivirale Starterpackung oder eine Verordnung mit den entsprechenden Anweisungen übergeben werden, damit er die Behandlung selbst so früh wie möglich einleiten kann (Tab. 4) [37-42] (C).

\subsubsection{Suppressive Behandlung}

Bei Patienten mit häufigen Rezidiven ( $>6$ pro Jahr) wurde eine Reduktion der Rezidivhäufigkeit um 70 bis $80 \%$ nachgewiesen [44] (I). Sicherheit und Wirksamkeit einer suppressiven Behandlung sind für Aciclovir für einen Zeitraum von mehr als 10 Jahren [45] und für Valaciclovir und Famciclovir für einen Zeitraum von über einem Jahr nachgewiesen [29, 44, 46-48]. Valaciclovir $2 \times 250 \mathrm{mg} / \mathrm{d}$ ist geringfügig wirksamer als $1 \times 500 \mathrm{mg} / \mathrm{d}$ und wird daher für Patienten mit mehr als 10 Rezidiven pro Jahr empfohlen [44] (A). Die suppressive Behandlung verbessert die Lebensqualität [49] (I). Mit den Jahren nimmt die Rezidivhäufigkeit auf unterschiedliche Weise ab [7]. Dies rechtfertigt, die suppressive Behandlung nach 6-12 Monaten für einen Zeitraum abzusetzen (d.h. zumindest zwei Rezidive abwarten), damit der natürliche Verlauf beurteilt 
werden kann [50] (B). Falls weiterhin häufige oder belastende Rezidive auftreten, ist es durchaus möglich, die suppressive Behandlung wieder aufzunehmen, die weder zu nennenswerten Nebenwirkungen noch zur Selektion resistenter Stämme führt [51] (IV).

Die suppressive Behandlung mit Valaciclovir bei Patienten mit Rezidiven bringt noch einen weiteren Nutzen: sie verringert die sexuelle Übertragung von HSV-2 bei Partnern mit unterschiedlichem serologischem Status um 50\%, die Übertragung von klinisch symptomatischen HSV-2Infektionen wird um $75 \%$ reduziert [12] (I).

\subsection{Behandlung bei immunsupprimierten Patienten}

Bei Immunsupprimierten und insbesondere bei fortgeschrittener HIV-Infektion beobachtet man chronische oder schwere Verläufe. Hier empfiehlt sich häufig eine episodische oder suppressive Behandlung [29] (B). In schweren Fällen
(z.B. bei viszeraler Dissemination) oder bei Unfähigkeit, Medikamente einzunehmen, kann die intravenöse Verabreichung notwendig sein (Tab. 4). Die Wahrscheinlichkeit der Selektion resistenter Stämme steigt mit dem Grad der Immunsuppression und der Anzahl der antiviralen Behandlungen. Sprechen die Läsionen nicht mehr auf die Standardbehandlung an, sollte ein Spezialist konsultiert werden (C).

\section{Information und Beratung des Patienten}

Information und Beratung der Patienten sind integrierter Bestandteil der Behandlung des Herpes genitalis und haben zwei Ziele: dem Patienten Hilfe anbieten, seine Krankheit zu verarbeiten, und die Vorbeugung der sexuellen und perinatalen Übertragung [59]. Im allgemeinen können Information und Beratung im Rahmen einer

Tabelle 4

Behandlung des Herpes genitalis.

\begin{tabular}{|c|c|c|}
\hline & Erstepisode & Rezidive \\
\hline \multicolumn{3}{|l|}{ Immunkompetente Personen } \\
\hline \multirow[t]{4}{*}{ Episodische Therapie } & Registrierungen in der Schweiz: & Registrierungen in der Schweiz: \\
\hline & $\begin{array}{l}\text { Aciclovir oral } 5 \times 200 \mathrm{mg} / \mathrm{d} \times 5-10 \mathrm{~d}(\mathrm{~A})[27,34] \\
\text { Valaciclovir } 2 \times 500 \mathrm{mg} / \mathrm{d} \times 5-10 \mathrm{~d}(\mathrm{~A}) \\
\text { Famciclovir } 3 \times 250 \mathrm{mg} / \mathrm{d} \times 5-10 \mathrm{~d}\left(\mathrm{C}^{*}\right) \\
\text { Aciclovir iv } 3 \times 5 \mathrm{mg} / \mathrm{kg} / \mathrm{d} \times 5 \mathrm{~d}(\mathrm{~A})[28,35]\end{array}$ & $\begin{array}{l}\text { Aciclovir oral } 5 \times 200 \mathrm{mg} / \mathrm{d} \times 5 \mathrm{~d}(\mathrm{~A})[38,39] \\
\text { Valaciclovir } 2 \times 500 \mathrm{mg} / \mathrm{d} \times 3-5 \mathrm{~d}(\mathrm{~A})[37,42] \\
\text { Famciclovir } 2 \times 125 \mathrm{mg} / \mathrm{d} \times 5 \mathrm{~d}(\mathrm{~A})[40]\end{array}$ \\
\hline & Andere Möglichkeiten: & Andere Möglichkeiten: \\
\hline & $\begin{array}{l}\text { Aciclovir oral } 3 \times 400 \mathrm{mg} / \mathrm{d} \times 7-10 \mathrm{~d}(\mathrm{C}) \\
\text { Valaciclovir } 2 \times 1000 \mathrm{mg} / \mathrm{d} \times 7-10 \mathrm{~d}(\mathrm{~A})[30]\end{array}$ & $\begin{array}{l}\text { Aciclovir oral } 3 \times 400 \mathrm{mg} / \mathrm{d} \times 5 \mathrm{~d}(\mathrm{C}) \\
\text { Aciclovir oral } 3 \times 800 \mathrm{mg} / \mathrm{d} \times 2 \mathrm{~d}(\mathrm{~A})[41]\end{array}$ \\
\hline $\begin{array}{l}\text { Suppressive Therapie } \\
\text { (empfohlen bei }>6 \text { Rezidiven/Jahr } \\
\text { oder starker Auswirkung } \\
\text { auf die Lebensqualität) }\end{array}$ & - & $\begin{array}{l}\text { Aciclovir oral } 2 \times 400 \mathrm{mg} / \mathrm{d}(\mathrm{A})[52,53] \\
\text { Valaciclovir } 2 \times 250 \mathrm{mg} / \mathrm{d}(>10 \text { Rezidive/Jahr }) \\
\text { oder } 1 \times 500 \mathrm{mg} / \mathrm{d}(<10 \text { Rezidive/Jahr })(\mathrm{A}) \text { [37] } \\
\text { Famciclovir } 2 \times 250 \mathrm{mg} / \mathrm{d}(\mathrm{A})[47,48]\end{array}$ \\
\hline \multicolumn{3}{|l|}{ Immunsupprimierte Personen } \\
\hline Episodische Therapie & Rücksprache mit Spezialisten (C) & $\begin{array}{l}\text { Aciclovir iv } 3 \times 5 \mathrm{mg} / \mathrm{kg} / \mathrm{d}(\mathrm{A}) \text { [54] } \\
\text { Aciclovir } 5 \times 400 \mathrm{mg} / \mathrm{d}(\mathrm{A}) \text { [55] } \\
\text { Valaciclovir } 2 \times 1000 \mathrm{mg} / \mathrm{d}(\mathrm{C}) \text { [56] } \\
\text { Famciclovir } 2 \times 500 \mathrm{mg} / \mathrm{d} \text { (A, AIDS-Patienten) [57] }\end{array}$ \\
\hline Suppressive Therapie & - & $\begin{array}{l}\text { Aciclovir } 2-3 \times 400-800 \mathrm{mg} / \mathrm{d}[54] \\
\text { Valaciclovir } 2 \times 500 \mathrm{mg} / \mathrm{d}(\mathrm{A})^{\star} \\
\text { Famciclovir } 2 \times 500 \mathrm{mg} / \mathrm{d}(\mathrm{A})[58]\end{array}$ \\
\hline Symptomatische Behandlung & $\begin{array}{l}\text { Analgetika, antiseptisch und antiphlogistisch wirkende } \\
\text { Externa (C) }\end{array}$ & $\begin{array}{l}\text { Analgetika, antiseptisch und antiphlogistisch } \\
\text { wirkende Externa (C) }\end{array}$ \\
\hline \multicolumn{3}{|l|}{ Schwangerschaft } \\
\hline Episodische Therapie & $\begin{array}{l}\text { Aciclovir oral } 5 \times 200 \mathrm{mg} / \mathrm{d} \times 10 \mathrm{~d} \\
\text { Valaciclovir } 2 \times 500 \mathrm{mg} / \mathrm{d} \times 10 \mathrm{~d}^{\circ}\end{array}$ & $\begin{array}{l}\text { Aciclovir oral } 5 \times 200 \mathrm{mg} / \mathrm{d} \times 5 \mathrm{~d} \\
\text { Valaciclovir } 2 \times 500 \mathrm{mg} / \mathrm{d} \times 5 \mathrm{~d}\end{array}$ \\
\hline $\begin{array}{l}\text { Suppressive Therapie (ab Woche } 36 \\
\text { bis zur Entbindung) }\end{array}$ & $\begin{array}{l}\text { Aciclovir oral } 3 \times 400 \mathrm{mg} / \mathrm{d} \\
\text { Valaciclovir } 2 \times 250 \mathrm{mg} / \mathrm{d}^{\circ}\end{array}$ & $\begin{array}{l}\text { Aciclovir oral } 3 \times 400 \mathrm{mg} / \mathrm{d} \\
\text { Valaciclovir } 2 \times 250 \mathrm{mg} / \mathrm{d}\end{array}$ \\
\hline
\end{tabular}


einzigen Konsultation nicht umfassend erfüllt werden. Viele Patienten können zusätzliche Informationsquellen nutzen: Drucksachen und Internetsites (z. B. www.herpesalliance.org, www. herpesnet.ch).

Die psychosoziale Belastung von Herpes ist nicht zu unterschätzen. Er wird oft als stigmatisierende Krankheit empfunden und wirkt sich negativ auf die Lebensqualität aus. Eine umfassende Information und Beratung kann die negativen psychischen Auswirkungen, die den rezidivierenden Herpes begleiten, vermindern [60] (B). Die Patienten sind auch $\mathrm{zu}$ informieren, dass HSV-2 bei der Pathogenese des Zervixkarzinoms keine ursächliche Rolle spielt (A).

Die mit dem Patienten zu besprechenden Punkte sind in Tabelle 5 zusammengefasst.

\section{Betreuung der Partner}

Auch eine Beratung der Sexualpartner ist indiziert. Symptomatische Partner werden in gleicher Weise wie der Indexpatient betreut. Asymptomatische Partner sollten auf Läsionen hin befragt und beraten werden, wie man Herpesläsionen erkennt. Ein typspezfischer serologischer Test sollte angeboten werden (C).

\section{Herpes genitalis während der Schwangerschaft}

\subsection{Vertikale Übertragung}

HSV-2 oder HSV-1 können unter der Geburt von der Mutter auf das Kind übertragen werden und beim Neugeborenen eine oft schwerverlaufende Infektion auslösen. Gemäss einer laufenden nationalen Beobachtungsstudie [61] kommt in der Schweiz die neonatale Herpesinfektion sehr selten vor.

Das perinatale Übertragungsrisiko hängt vom Charakter der aktuellen Episode (Primärinfektion $>>$ Reaktivierung) und vom Virustyp (HSV$1>$ HSV-2) ab. Es kann bei positivem Virusnachweis zum Zeitpunkt der Geburt zwischen 30\% bei Primärinfektionen und $<1 \%$ bei Rezidiven variieren $[62,63]$. Die Sectio caesarea reduziert das Übertragungsrisiko (OR 0,14, 95\%; CI 0,02-1,08) [62] (III).

\subsection{Abschätzung des Risikos für das Neugeborene}

Obwohl für die Beurteilung des Risikos für das Neugeborene äusserst wichtig, ist die klinische Unterscheidung zwischen Primärinfektion, Initialinfektion mit dem anderen HSV-Typ und
Reaktivierung in der Schwangerschaft nicht möglich (III). Eine erste Manifestation von Herpes genitalis während der Schwangerschaft ist in den meisten Fällen keine Primärinfektion [64]. Umgekehrt sind die meisten Primärinfektionen nicht symptomatisch [63]. Zudem kann eine klinisch als Rezidiv erscheinende Episode durchaus auch eine Initialinfektion mit dem anderen Serotyp und damit ebenfalls von markant erhöhtem neonatalem Risiko sein [62]. Aufgrund dieser Daten erscheint ein unterschiedliches Management erstmaliger und rezidivierender Episoden von Herpes genitalis in der Schwangerschaft nicht sinnvoll. Dies ändert sich, wenn die Art der Infektion durch Virustypisierung und Bestimmung der typspezifischen Antikörper charakterisiert werden kann.

Weder ein serologisches noch ein virologisches (Kultur oder PCR) Screening vor der Geburt erlauben eine zuverlässige Erfassung der von einer neonatalen Herpesinfektion bedrohten Neugeborenen [62] (III). Ein solches Screening wird deshalb nicht empfohlen (D). Der Stellenwert eines serologischen Screenings zu Beginn der Schwangerschaft, um Frauen mit einem Risiko für eine Primärinfektion zu identifizieren, wird momentan untersucht.

\subsection{Management schwangerer Frauen mit einer ersten oder rezidivierenden Herpes- genitalis-Episode}

\subsubsection{Behandlung klinischer Episoden}

Schwangere mit einer ersten klinischen Episode oder einem Rezidiv können mit Aciclovir oder Valaciclovir in den empfohlenen Dosierungen (Tab. 4) ohne unangemessenes Risiko für den Fötus behandelt werden $[65,66]$.

\subsubsection{Suppressive Behandlung}

Randomisierte Studien haben gezeigt, dass eine suppressive Behandlung ab der 36. Schwangerschaftswoche bis zur Entbindung die Häufigkeit klinischer Manifestationen sowie der Virusausscheidung zum Zeitpunkt der Geburt verringert und sie damit oft eine vaginale Geburt erlauben kann [67-70] (I) (A).

\subsubsection{Geburtsmodus}

Eine positive Anamnese für Herpes genitalis bei fehlenden genitalen Symptomen (aktive Läsionen oder prodromale Schmerzen oder Brennen) zum Geburtsbeginn ist keine Indikation für eine Sectio caesarea [70] (E). Wenn bei der Präsentation zur Geburt klinische Herpesläsionen und/ oder ein positiver Virusnachweis vorliegen, ist ein Kaiserschnitt eine wirksame Präventions- 
Tabelle 5

Checkliste zur Patientenberatung.

\begin{tabular}{|c|c|}
\hline Thema & Vorgehen \\
\hline \multicolumn{2}{|l|}{ Natürlicher Verlauf und Epidemiologie } \\
\hline \multirow[t]{4}{*}{ Verlauf und Formen der Erkrankung } & $\begin{array}{l}\text { Das Rezidivpotential, die asymptomatische Virusausscheidung und das } \\
\text { Übertragungsrisiko erklären }\end{array}$ \\
\hline & $\begin{array}{l}\text { Aufzeigen, dass Herpes genitalis durch HSV-2 oder durch HSV-1 verursacht } \\
\text { werden kann }\end{array}$ \\
\hline & Erwähnen, dass weltweit eine von sechs Personen infiziert ist \\
\hline & $\begin{array}{l}\text { Betonen, dass } 80 \% \text { der Infektionen nicht erkannt sind, weil die Symptome } \\
\text { minimal sind oder gänzlich fehlen }\end{array}$ \\
\hline \multirow[t]{3}{*}{ Information der Sexualpartner } & $\begin{array}{l}\text { Den Patienten / die Patientin motivieren, gegenwärtige und zukünftige } \\
\text { Sexualpartner über seinen/ihren Infektionsstatus zu informieren }\end{array}$ \\
\hline & $\begin{array}{l}\text { Sexualpartner von infizierten Personen sollten aufgeklärt werden, dass sie } \\
\text { infiziert sein können, ohne je Symptome gehabt zu haben }\end{array}$ \\
\hline & $\begin{array}{l}\text { Eine typspezifische serologische Abklärung kann bei der Beratung über } \\
\text { die notwendigen Vorsichtsmassnahmen hilfreich sein }\end{array}$ \\
\hline \multirow[t]{3}{*}{ Asymptomatische Virusausscheidung } & $\begin{array}{l}\text { Erklären, dass die asymptomatische Virusausscheidung mehrheitlich für die } \\
\text { Ansteckung verantwortlich ist }\end{array}$ \\
\hline & $\begin{array}{l}\text { Sie erfolgt häufiger im ersten Jahr nach der Primärinfektion, bei Immun- } \\
\text { supprimierten und bei Patienten mit häufigen Rezidiven ( }>12 / J a h r)\end{array}$ \\
\hline & HSV-2 wird genital häufiger asymptomatisch ausgeschieden als HSV-1 \\
\hline \multirow[t]{3}{*}{ Kontaktübertragung } & $\begin{array}{l}\text { Erklären, dass die Übertragung durch Hautkontakt genital erfolgt (auch } \\
\text { bei fehlender Penetration, z. B. beim «Petting») }\end{array}$ \\
\hline & $\begin{array}{l}\text { Die Übertragung kann auch orogenital erfolgen. Prinzipiell können durch } \\
\text { Verschleppung sämtliche Körperregionen betroffen sein }\end{array}$ \\
\hline & $\begin{array}{l}\text { Bei Vorliegen von Prodromi oder Läsionen ist die Virusausscheidung } \\
\text { praktisch sicher, weshalb dann auf sexuelle Kontakte mit nichtinfizierten } \\
\text { Partnern verzichtet werden soll }\end{array}$ \\
\hline Gebrauch von Präservativen & $\begin{array}{l}\text { Erklären, dass Latexpräservative das Infektionsrisiko von Mann zu Frau } \\
\text { verringern können }\end{array}$ \\
\hline \multirow[t]{3}{*}{ Neonataler Herpes } & $\begin{array}{l}\text { Herpes neonatorum als schwerwiegendste, aber seltene Komplikation } \\
\text { muss allen Betroffenen erklärt werden }\end{array}$ \\
\hline & $\begin{array}{l}\text { Schwangere Frauen oder Frauen im gebärfähigen Alter, die mit HSV-2 } \\
\text { infiziert sind, müssen die Geburtshelfer sowie den Pädiater ihres Kindes } \\
\text { darüber informieren }\end{array}$ \\
\hline & $\begin{array}{l}\text { Nicht mit HSV-2 infizierte Schwangere müssen während der Schwanger- } \\
\text { schaft (speziell im letzten Trimenon) auf die Gefahr von Sexualkontakten } \\
\text { (genital und orogenital) mit einem infizierten Partner aufmerksam ge- } \\
\text { macht werden }\end{array}$ \\
\hline Atypische Symptome & $\begin{array}{l}\text { Asymptomatische Personen, bei denen mittels typspezifischer Serologie } \\
\text { eine Infektion mit HSV-2 diagnostiziert wurde, müssen beraten und die } \\
\text { möglichen Hautmanifestationen (inkl. atypischer Formen) erklärt werden. }\end{array}$ \\
\hline \multicolumn{2}{|l|}{ Mögliche Therapien } \\
\hline Optionen & $\begin{array}{l}\text { Möglichkeit und Wirkung der Therapieformen erläutern (episodische anti- } \\
\text { virale Therapie, Suppressionstherapie, unspezifische Lokaltherapie) }\end{array}$ \\
\hline \multirow[t]{4}{*}{ Suppressionstherapie } & $\begin{array}{l}\text { Erklären, dass es auch unter einer Suppressionstherapie vereinzelt zu } \\
\text { (meist wenige Tage dauernden) Rezidiven kommen kann }\end{array}$ \\
\hline & $\begin{array}{l}\text { In diesem Fall erhöht man die Dosis vorübergehend (Tab. 4; entsprechend } \\
\text { der episodischen Behandlung) }\end{array}$ \\
\hline & $\begin{array}{l}\text { Betonen, dass die Suppressionstherapie den natürlichen Verlauf der Infek- } \\
\text { tion nicht verändert. Nach deren Absetzen ist mit Rezidiven zu rechnen }\end{array}$ \\
\hline & $\begin{array}{l}\text { Da in der Regel mit zunehmendem Alter die Rezidivhäufigkeit abnimmt, } \\
\text { kann je nach Dauer der Therapie mit weniger Rezidiven gerechnet werden. } \\
\text { Im Fall des Wiederauftretens häufiger Rezidive kann die suppressive Be- } \\
\text { handlung wieder aufgenommen werden }\end{array}$ \\
\hline Wirkung auf Virusausscheidung & $\begin{array}{l}\text { Informieren, dass die suppressive Behandlung die Virusausscheidung } \\
\text { signifikant vermindert und das Risiko einer Virusübertragung um } 50 \% \text { re- } \\
\text { duziert [12] (I) }\end{array}$ \\
\hline Verträglichkeit & $\begin{array}{l}\text { Dem Patienten versichern, dass unerwünschte Wirkungen der suppressi- } \\
\text { ven Behandlung äusserst selten sind, und dass bei immunkompetenten } \\
\text { Patienten keine Resistenzprobleme beobachtet werden }\end{array}$ \\
\hline
\end{tabular}


massnahme [62] (III) und deshalb indiziert (B). Bei Blasensprung sollte, sofern die fötale Lungenreife anzunehmen ist, der Kaiserschnitt möglichst rasch, spätestens innert 4-6 Stunden, durchgeführt werden [69] (B).

Bei unreifer Lunge gibt es keine gesicherten Grundlagen für die Entscheidung. Das geringe Risiko einer manifesten fötalen/neonatalen Herpesinfektion trotz mütterlicher antiviraler Therapie bei fortgeführter Schwangerschaft (mit oder ohne Lungenreifungsinduktion durch Kortikosteroide) muss gegen das besser abschätzbare (v.a. vom Gestationsalter abhängige) Risiko der sofortigen Entbindung durch Kaiserschnitt abgewogen werden [69]. Das Risiko eines expektativen Vorgehens bei Schwangeren mit rezidivierendem Herpes genitalis und vorzeitigem Blasensprung scheint gering zu sein [70] (IV) (B). In diesen komplexen Fällen ist die sofortige Verlegung in ein Zentrum zu empfehlen (C).

Bei intakter Fruchtblase und fehlenden Wehen ist ein Herpes genitalis bis kurz vor dem errechneten Termin nie eine alleinige Indikation für eine primäre Sectio caesarea [70] (D). Es gilt zu beachten, dass auch mit 37 und 38 Schwangerschaftswochen noch ein relevantes Risiko eines neonatalen Atemnotsyndroms besteht, das bei Geburt durch elektiven Kaiserschnitt zusätzlich stark erhöht wird [71] (III).

Nach einem klinischen Rezidiv während der Schwangerschaft ist eine vaginale Geburt möglich, sobald und solange keine klinischen Läsionen mehr vorhanden sind. Wenn allerdings eine Primärinfektion oder eine Initialinfektion mit dem anderen HSV-Typ nachgewiesen ist oder sehr wahrscheinlich erscheint, so ist die optimale Vorgehensweise nicht genau definiert. Die meisten Guidelines empfehlen eine Sectio caesarea bei allen Frauen, die 4-6 Wochen vor der Geburt eine klinische Erstmanifestation einer Herpesinfektion entwickeln [1, 72-76] (IV) (C). Einige Experten denken jedoch, dass der Fötus bei einer vaginalen Geburt bis zum Erscheinen von typspezifischen Antikörpern einem Risiko ausgesetzt ist, was 8-12 Wochen dauern kann [77] (C).

\section{Neonatale Herpes-simplex-Virus- Infektion}

\subsection{Klinik}

Die neonatale Herpesinfektion manifestiert sich unter drei klinisch unterschiedlichen Bildern. Die häufigste (50\% der Fälle) und mildeste Form bleibt lokalisiert auf Haut und/oder Augen bzw. Mund. Der vorwiegende Befall des ZNS, die neonatale Herpesenzephalitis (33\%), manifestiert sich mit Lethargie und Krämpfen und führt sehr häufig zu bleibenden Spätfolgen. Die disseminierte Form (17\%) hat eine hohe Letalität und geht mit Hepatitis, Pneumonie, disseminierter intravasaler Gerinnung und Schock einher [78].

Im Moment der Diagnosestellung finden sich die Symptome mit folgender Häufigkeit: Bläschen auf der Haut 68\%, Fieber 39\%, Lethargie $38 \%$, Krämpfe $27 \%$, Konjunktivitis $19 \%$, Pneumonie $13 \%$, disseminierte intravasale Gerinnung $11 \%$.

Symptome können selten bereits bei Geburt vorliegen, erscheinen jedoch in $60 \%$ der Fälle später als 5 Tage nach der Geburt und treten manchmal erstmals 4-6 Wochen postnatal auf [79] (III).

\subsection{Diagnostisches Vorgehen}

\subsubsection{Bei bekannter perinataler HSV-Exposition}

Bei Vorliegen eines manifesten Herpes genitalis unter der Geburt oder bekannter subpartaler Virusausscheidung der Mutter wird beim Neugeborenen die Kultivierung von Abstrichen von Konjunktiven, Oropharynx und Rektum empfohlen. Um eine Übertragung (nicht bloss Kontamination) nachzuweisen, sollten die Abstriche im Alter von 24 bis 48 Stunden abgenommen werden [69] (C).

Das Neugeborene muss sorgfältig und genügend lange (bis zum Alter von 4 bis 6 Wochen) auf allenfalls auftretende Symptome einer neonatalen Herpesinfektion überwacht werden. Bei der Spitalentlassung müssen die Eltern entsprechend instruiert werden (B).

\subsubsection{Bei herpesverdächtigen Symptomen}

Da der grösste Teil der neonatalen Herpesinfektionen nach negativer mütterlicher Anamnese für Herpes genitalis auftritt [63], muss bei allen Neugeborenen mit entsprechenden Symptomen unverzüglich nach einer HSV-Infektion gesucht werden (B). Ein Drittel der Neugeborenen mit Herpesinfektion zeigt keine charakteristischen Hautläsionen und stellt deshalb eine besondere diagnostische Herausforderung dar [79].

An eine neonatale Herpesinfektion muss insbesondere gedacht werden bei:

- charakteristischen Haut- oder Schleimhautläsionen;

- Konjunktivitis, insbesondere bei Injektion der Conjunctiva bulbi, bzw. Keratitis;

- Krämpfen und/oder Lethargie ohne andere Erklärung;

- Fieber oder anderen systemischen Symptomen ohne andere Erklärung. 
Bei Verdacht auf neonatale HSV-Infektion sind folgende Untersuchungen durchzuführen [62] (C):

- Kultur von Bläschen, Konjunktiven, Oropharynx, Stuhl/Rektum, Urin, Blut;

- Lumbalpunktion mit HSV-PCR (die Kultur aus Liquor ist wenig sensitiv);

- Routinelabor inkl. Transaminasen und Gerinnungsuntersuchung;

- zerebrale Bildgebung und/oder ophthalmologische Untersuchung wenn indiziert.

\subsection{Therapie und Prognose}

Die genügend lange, hochdosierte Therapie mit intravenösem Aciclovir hat eine gesicherte Wirksamkeit [78] (II) bei allerdings noch erheblicher Restletalität und -morbidität. Während die lokalisierte Form immer folgenlos ausheilt, bringt die ZNS-Erkrankung eine Letalität von 6\% und bleibende Spätfolgen in $69 \%$ mit sich. Die disseminierte Infektion verläuft in 31\% tödlich und führt bei $17 \%$ zu Spätfolgen [78]. Entscheidend für die Prognose ist der Zeitpunkt des Einsetzens der Therapie im Krankheitsverlauf (III).

Zur Erreichung optimaler Therapieresultate muss bei jedem klinischen Verdacht auf neonatale Herpesinfektion sofort nach Abnahme der im vorhergehenden Kapitel beschriebenen diagnostischen Proben mit einer intravenösen Therapie mit Aciclovir $60 \mathrm{mg} / \mathrm{kg} / \mathrm{d}$ in drei Gaben begonnen werden (A). Bei negativen virologischen Untersuchungen kann die Therapie wieder abgesetzt werden. Bei lokalisierter HSV-Infektion beträgt die Therapiedauer 14, bei ZNS-Erkrankung oder disseminierter Infektion 21 Tage [78] (B). Bei Augenbeteiligung soll zusätzlich eine lokale antivirale Therapie appliziert werden.

Nach einer neonatalen Herpesinfektion können kutane Rezidive über längere Zeit auftreten. Die Rolle einer suppressiven Therapie für diese Fälle ist noch nicht geklärt. Bei Herpesinfektion des Auges soll 2-3 Wochen nach Absetzen der Therapie eine ophthalmologische Untersuchung zum Ausschluss einer Keratitis durchgeführt werden.

Eine präemptive Aciclovirtherapie bei asymptomatischen Neugeborenen von Müttern mit Herpes genitalis hat keine gesicherte Grundlage und wird nicht empfohlen [69] (D).

\subsection{Präventive Massnahmen}

Neugeborene mit nachgewiesener oder vermuteter HSV-Infektion sollen isoliert und unter Vermeidung des Direktkontakts mit Haut- und Schleimhautläsionen sowie Ausscheidungen und Körperflüssigkeiten gepflegt werden.
Herpesläsionen an Lippen, Fingern oder Brustwarzen der Mutter oder anderer Personen mit direktem Kontakt mit Neugeborenen können ein - allerdings geringes - Risiko einer nosokomialen Übertragung auf andere Neugeborene darstellen. Mütter, Väter, Besucher und medizinisches Personal der Wochenbettstation müssen darüber unterrichtet werden und die notwendigen Vorsichtsmassnahmen (dichtes Abdecken der Läsionen zur Vermeidung eines direkten Kontaktes mit Haut oder Schleimhäuten des Neugeborenen) treffen. Medizinisches Personal mit Herpes labialis muss bei Einhaltung dieser Vorsichtsmassnahmen nicht von der Betreuung Neugeborener suspendiert werden [69] (B).

\section{Ophthalmologie}

Wegen des hohen Komplikationsrisikos auch «einfacher» epithelialer Herpesinfektionen der Hornhaut und eines extrem hohen Risikos schwerer bleibender Sehbeeinträchtigungen bei Beteiligung tieferer Gewebsschichten sollten alle Patienten mit Augenbeteiligung in augenfachärztliche Kontrolle überwiesen werden.

\section{Literatur}

1 Patel R, Barton SE, Brown D, Cowan FM, Kinghorn GR, Munday PE, et al. European guideline for the management of genital herpes. Int J STD AIDS 2001;12(Suppl 3):34-39.

2 Workowski KA, Levine WC, Wasserheit JN. U.S. Centers for disease control and prevention guidelines for the treatment of sexually transmitted diseases: an opportunity to unify clinical and public health practice. Ann Intern Med 2002; 137(4):255-62.

3 Langenberg AG, Corey L, Ashley RL, Leong WP, Straus SE. A prospective study of new infections with herpes simplex virus type 1 and type 2 . Chiron HSV Vaccine Study Group. N Engl J Med 1999;341(19):1432-8.

4 Diamond C, Selke S, Ashley R, Benedetti J, Corey L. Clinical course of patients with serologic evidence of recurrent genital herpes presenting with signs and symptoms of first episode disease. Sex Transm Dis 1999;26:221-5.

5 Lowhagen GB, Tunback P, Andersson K, Bergstrom T, Johannisson G. First episodes of genital herpes in a Swedish STD population: a study of epidemiology and transmission by the use of herpes simplex virus (HSV) typing and specific serology. Sex Transm Infec 2000;76(3):179-82.

6 Jolivet P, Meylan PRA. Herpès génital: l'épidémie silencieuse? Méd Hyg 2001;59:781-9. 
7 Benedetti JK, Zeh J, Corey L. Clinical reactivation of genital herpes simplex virus infection decreases in frequency over time. Ann Intern Med 1999; 131(1):14-20

8 Buenzli D, Wietlisbach V, Barazzoni F, Sahli R, Meylan P. Seroepidemiology of herpes simplex virus type 1 and 2 in western and southern Switzerland in adults aged 25-74 in 1992-93: a population-based study. BMC Infect Dis 2004; 4:10.

9 Mertz GJ, Benedetti J, Ashley R, Selke SA, Corey L. Risk factors for the sexual transmission of genital herpes. Ann Intern Med 1992;116(3):197-202.

10 Brown ZA, Selke S, Zeh J, Kopelman J, Maslow A, Ashley RL, et al. The acquisition of herpes simplex virus during pregnancy. N Engl J Med 1997; 337:509-15.

11 Wald A, Langenberg AG, Link K, Izu AE, Ashley R, Warren $\mathrm{T}$, et al. Effect of condoms on reducing the transmission of herpes simplex virus type 2 from men to women. JAMA 2001;285(24):3100-6

12 Corey L, Wald A, Patel R, Sacks SL, Tyring SK, Warren T, et al. Once-daily valaciclovir to reduce the risk of transmission of genital herpes. N Engl J Med 2004;350:11-20.

13 Lafferty WE, Downey L, Celum C, Wald A. Herpes simplex virus type 1 as a cause of genital herpes: impact on surveillance and prevention. J Infect Dis 2000;181(4):1454-7.

14 Wald A, Link K. Risk of human immunodeficiency virus infection in herpes simplex virus type 2-seropositive persons: a meta-analysis. J Infect Dis 2002;185(1):45-52.

15 Corey L, Adams HG, Brown ZA, Holmes KK. Genital herpes simplex virus infections: clinical manifestations, course, and complications. Ann Intern Med 1983;98:958-72.

16 Cowan FM, Johnson AM, Ashley R, Corey L, Mindel A. Antibody to herpes simplex virus type 2 as serological marker of sexual lifestyle in populations. Br Med J 1994;309(6965):1325-9.

17 Benedetti J, Corey L, Ashley R. Recurrence rates in genital herpes after symptomatic first-episode infection. Ann Intern Med 1994;121(11):847-54.

18 Lafferty WE, Coombs RW, Benedetti J, Critchlow C, Corey L. Recurrences after oral and genital herpes simplex virus infection. Influence of site of infection and viral type. N Engl J Med 1987;316:1444-9.

19 Fleming DT, McQuillan GM, Johnson RE, Nahmias AJ, Aral SO, Lee FK, et al. Herpes simplex virus type 2 in the United States, 1976 to 1994. N Engl J Med 1997;337(16):1105-11.

20 Langenberg A, Benedetti J, Jenkins J, Ashley R, Winter C, Corey L. Development of clinically recognizable genital lesions among women previously identified as having «asymptomatic» herpes simplex virus type 2 infection. Ann Intern Med 1989;110(11):882-7.

21 Lautenschlager S, Eichmann A. The heterogeneous clinical spectrum of genital herpes. Dermatology 2001;202(3):211-9.
22 Slomka MJ, Emery L, Munday PE, Moulsdale M, Brown DW. A comparison of PCR with virus isolation and direct antigen detection for diagnosis and typing of genital herpes. J Med Virol 1998; 55(2):177-83.

23 Coyle PV, Desai A, Wyatt D, McCaughey C, $\mathrm{O}^{\prime}$ Neill HJ. A comparison of virus isolation, indirect immunofluorescence and nested multiplex polymerase chain reaction for the diagnosis of primary and recurrent herpes simplex type 1 and type 2 infections. J Virol Methods 1999; 83(1-2):75-82.

24 Scoular A, Gillespie G, Carman WF. Polymerase chain reaction for diagnosis of genital herpes in a genitourinary medicine clinic. Sex Transm Infect 2002;78(1):21-5.

25 Schmid DS, Brown DR, Nisenbaum R, Burke RL, Alexander D, Ashley R, et al. Limits in reliability of glycoprotein G-based type specific serologic assays for herpes simplex virus types 1 and 2 . J Clin Microbiol 1999;37:376-9.

26 Corey L, Nahmias AJ, Guinan ME, Benedetti JK, Critchlow CW, Holmes KK. A trial of topical acyclovir in genital herpes simplex virus infections. N Engl J Med 1982;306(22):1313-9.

27 Bryson YJ, Dillon M, Lovett M, Acuna G, Taylor S Cherry JD, et al. Treatment of first episodes of genital herpes simplex virus infection with oral acyclovir. A randomized double-blind controlled trial in normal subjects. N Engl J Med 1983; 308(16):916-21.

28 Mindel A, Adler MW, Sutherland S, Fiddian AP. Intravenous acyclovir treatment for primary genital herpes. Lancet 1982;1(8274):697-700.

29 Wald A. New therapies and prevention strategies for genital herpes. Clin Infect Dis 1999; 28(Suppl 1):S4-13.

30 Fife KH, Barbarash RA, Rudolph T, Degregorio B, Roth R. Valaciclovir versus acyclovir in the treatment of first-episode genital herpes infection. Results of an international, multicenter, doubleblind, randomized clinical trial. The Valaciclovir International Herpes Simplex Virus Study Group. Sex Transm Dis 1997;24(8):481-6.

31 Patel R. Progress in meeting today's demands in genital herpes: an overview of current management. J Infect Dis 2002;186(Suppl 1):S47-56.

32 Garcia A, Garcia S, Schanchez JA, Garcia I, Lanchares JL. Valaciclovir in the treatment of initial infection by genital herpes virus: comparative study. Enferm Infecc Microbiol Cin 2001;19(1): $15-8$.

33 Corey L, Mindel A, Fife KH, Sutherland S, Benedetti J, Adler MW. Risk of recurrence after treatment of first-episode genital herpes with intravenous acyclovir. Sex Transm Dis 1985;12(4): 215-8.

34 Mertz GJ, Critchlow CW, Benedetti J, Reichman RC, Dolin R, Connor J, et al. Double-blind placebocontrolled trial of oral acyclovir in first-episode genital herpes simplex virus infection. JAMA 1984;252(9):1147-51 
35 Peacock JE Jr, Kaplowitz LG, Sparling PF, Durack DT, Gnann JW Jr, Whitley RJ, et al. Intravenous acyclovir therapy of first episodes of genital herpes: a multicenter double-blind, placebo-controlled trial. Am J Med 1988;85(3):301-6.

36 Bodsworth NJ, Crooks RJ, Borelli S, Vejlsgaard G, Paavonen J, Worm AM, et al. Valaciclovir versus aciclovir in patient initiated treatment of recurrent genital herpes: a randomised, double blind clinical trial. International Valaciclovir HSV Study Group. Genitourin Med 1997;73(2):1510-6.

37 Spruance SL, Tyring SK, DeGregorio B, Miller C, Beutner K. A large-scale, placebo-controlled, doseranging trial of peroral valaciclovir for episodic treatment of recurrent herpes genitalis. Valaciclovir HSV Study Group. Arch Intern Med 1996; 156(15):1729-35.

38 Nilsen AE, Aasen T, Halsos AM, Kinge BR, Tjotta EA, Wikstrom K, et al. Efficacy of oral acyclovir in the treatment of initial and recurrent genital herpes. Lancet 1982;2(8298):571-3.

39 Reichman RC, Badger GJ, Mertz GJ, Corey L, Richman DD, Connor JD, et al. Treatment of recurrent genital herpes simplex infections with oral acyclovir. A controlled trial. JAMA 1984;251(16): 2103-7.

40 Sacks SL, Aoki FY, Diaz-Mitoma F, Sellors J, Shafran SD. Patient-initiated, twice-daily oral famciclovir for early recurrent genital herpes. A randomized, double-blind multicenter trial. Canadian Famciclovir Study Group. JAMA 1996; 276(1):44-9.

41 Wald A, Carrell D, Remington M, Kexel E, Zeh J, Corey L. Two-day regimen of acyclovir for treatment of recurrent genital herpes simplex virus type 2 infection. Clin Infect Dis 2002;34(7):944-8

42 Leone PA, Trottier S, Miller JM. Valacyclovir for episodic treatment of genital herpes: a shorter 3-day treatment course compared with 5-day treatment. Clin Infect Dis 2002;34(7):958-6.

43 Fife KH, Almekinder J, Madhura BP, Deeter RG. Episodic versus suppressive therapy for recurrent genital herpes. $41^{\text {st }}$ ICAAC, Chicago, 2001.

44 Reitano M, Tyring S, Lang W, Thoming C, Worm AM, Borelli S, et al. Valaciclovir for the suppression of recurrent genital herpes simplex virus infection: a large-scale dose range-finding study. International Valaciclovir HSV Study Group. J Infect Dis 1998;178(3):603-10.

45 Goldberg LH, Kaufman R, Kurtz TO, Conant MA, Eron LJ, Batenhorst RL. Long-term suppression of recurrent genital herpes with acyclovir. A 5-year benchmark. Acyclovir Study Group. Arch Dermatol 1993;129(5):582-7.

46 Chosidow O, Drouault Y, Leconte-Veyriac F, Aymard M, Ortonne JP, et al. Famciclovir vs. aciclovir in immunocompetent patients with recurrent genital herpes infections: a parallel-groups, randomized, double-blind clinical trial. Br J Dermatol 2001;144(4):818-24.

47 Diaz-Mitoma F, Sibbald RG, Shafran SD, Boon R, Saltzman RL. Oral famciclovir for the suppression of recurrent genital herpes: a randomized controlled trial. Collaborative Famciclovir Genital Herpes Research Group. JAMA 1998;280(10): 887-92.
48 Mertz GJ, Loveless MO, Levin MJ, Kraus SJ, Fowler SL, Goade D, et al. Oral famciclovir for suppression of recurrent genital herpes simplex virus infection in women. A multicenter, doubleblind, placebo-controlled trial. Collaborative Famciclovir Genital Herpes Research Group. Arch Intern Med 1997;157(3):343-9.

49 Patel R, Tyring S, Strand A, Price MJ, Grant DM. Impact of suppressive antiviral therapy on the health-related quality of life of patients with recurrent genital herpes infection. Sex Transm Infect 1999;75(6):398-402.

50 Straus SE, Croen KD, Sawyer MH, Freifeld AG, Felser JM, Dale JK. Acyclovir suppression of frequently recurring genital herpes. Efficacy and diminishing need during successive years of treatment. JAMA 1988;260(15):2227-30.

51 Tyring SK, Baker D, Snowden W. Valacyclovir for herpes simplex virus infection: long-term safety and sustained efficacy after 20 years' experience with acyclovir. J Infect Dis 2002;186(Suppl 1): S40-46.

52 Mindel A, Faherty A, Carney O, Patou G, Freris M, Williams P. Dosage and safety of long-term suppressive acyclovir therapy for recurrent genital herpes. Lancet 1988;1(8591):926-8.

53 Mertz GJ, Jones CC, Mills J, Fife KH, Lemon SM, Stapleton JT, et al. Long-term acyclovir suppression of frequently recurring genital herpes simplex virus infection. A multicenter double-blind trial. JAMA 1988;260(2):201-6.

54 Wade JC, Newton B, McLaren C, Flournoy N, Keeney RE, Meyers JD. Intravenous acyclovir to treat mucocutaneous herpes simplex virus infection after marrow transplantation: a double-blind trial. Ann Intern Med 1982;96(3):265-9.

55 Shepp DH, Newton BA, Dandliker PS, Flournoy N Meyers JD. Oral acyclovir therapy for mucocutaneous herpes simplex virus infections in immunocompromised marrow transplant recipients. Ann Intern Med 1985;102(6):783-5.

56 Conant MA, Schacker TW, Murphy RL, Gold J, Crutchfield LT, Crooks RJ. Valaciclovir versus aciclovir for herpes simplex virus infections in HIV-infected individuals: two randomized trials. Int J STD AIDS 2002;13(1):12-21.

57 Romanowski B, Aoki FY, Martel AY, Lavender EA, Parsons JE, Saltzman RL. Efficacy and safety of famciclovir for treating mucocutaneous herpes simplex infection in HIV-infected individuals. Collaborative Famciclovir HIV Study Group. AIDS 2000;14(9):1211-7.

58 Schacker T, Hu HL, Koelle DM, Zeh J, Saltzman R, Boon R, et al. Famciclovir for the suppression of symptomatic and asymptomatic herpes simplex virus reactivation in HIV-infected persons. A double-blind, placebo-controlled trial. Ann Intern Med 1998;128(1):21-8.

59 Corey L. Challenges in genital herpes simplex virus management. J Infect Dis 2002;186(Suppl 1):S29-33.

60 Green J, Kocsis A. Psychological factors in recurrent genital herpes. Genitourin Med 1997;73(4): 253-8. 
61 Meylan P. Neonataler Herpes. In: SPSU - Jahresbericht 2002. BAG Bulletin 2003;37:643.

62 Brown ZA, Wald A, Morrow RA, Selke S, Zeh J, Corey L. Effect of serologic status and cesarean section on transmission rates of herpes simplex virus from mother to infant. JAMA 2003;289:203-9.

63 Brown ZA, Benedetti J, Ashley R, Burchett S, Selke S, Berry $S$, et al. Neonatal herpes simplex virus infection in relation to asymptomatic maternal infection at the time of labor. N Engl J Med 1991;324:1247-52.

64 Hensleigh PA, Andrews WW, Brown Z Greenspoon J, Yasukawa L, Prober CG. Genital herpes during pregnancy: inability to distinguish primary and recurrent infections clinically. Obstet Gynecol 1997;89:891-5.

65 Briggs GG, Freeman RK, Yaffe SJ. A Reference Guide to Fetal and Neonatal Risk: Drugs in Pregnancy and Lactation. $6^{\text {th }}$ edition. Philadelphia: Lippincott Williams \& Wilkins; 2002.

66 Reiff-Eldridge RA, Heffner CR, Ephross SA, Tennis PS, White AD, Andres EB. Monitoring pregnancy outcomes after prenatal drug exposure through prospective pregnancy registries: a pharmaceutical company commitment. Am J Obstet Gynecol 2000;182:159-63.

67 Sheffield JS, Hollier LM, Hill JB, Stuart GS, Wendel GD. Acyclovir prophylaxis to prevent herpes simplex virus recurrence at delivery: a systematic review. Obstet Gynecol 2003;102:1396-402.

68 Watts DH, Brown ZA, Money D, Selke S, Huang ML, Sacks SL, et al. A double-blind, randomized, placebo-controlled trial of acyclovir in late pregnancy for the reduction of herpes simplex virus shedding and cesarean delivery. Am J Obstet Gynecol 2003;188:836-43.

69 American Academy of Pediatrics. Herpes simplex. In: Pickering LK (ed.). Red Book: 2003 Report of the Committee on Infectious Diseases. $26^{\text {th }}$ edition. Elk Grove Village: American Academy of Pediatrics; 2003. p. 344-53.

70 Major CA, Towers CV, Lewis DF, Garite TJ. Expectant management of preterm premature rupture of membranes complicated by active recurrent genital herpes. Am J Obstet Gynecol 2003;188: 1551-5.

71 Morrison JJ, Rennie JM, Milton PJ. Neonatal respiratory morbidity and mode of delivery at term: influence of timing of elective caesarean section. Br J Obstet Gynaecol 1995;102:101-6.

72 Lecat M. Herpès cutanéo-muqueux chez l'immunocompétent et grossesse. Ann Dermatol Venereol 2002;129:523-32.

73 National guideline for the management of genital herpes. Sex Transm Infect 1999;75:S24-8.
74 Huraux JM. Mesure préventive de l'herpès du nouveau-né: conférence de consensus de 1993 et nouvelles propositions. Ann Dermatol Venerol 2002;129:646-51.

75 Randolph AG, Washington AE, Prober CG. Cesarean delivery for women presenting with genital herpes lesions. Efficacy, risks, and costs. JAMA 1993;270:77-82.

76 ACOG practice bulletin. Management of herpes in pregnancy. Number 8 October 1999. Clinical management guidelines for obstetrician-gynecologists. Int J Gynaecol Obstet 2000;68:165-73.

77 Brown ZA. Case study: type-specific HSV serology and the correct diagnosis of first-episode genital herpes during pregnancy. Herpes 2002;9:24-6.

78 Kimberlin DW, Lin CY, Jacobs RF, Powell DA, Corey L, Gruber WC, et al. Safety and efficacy of high-dose intravenous acyclovir in the management of neonatal herpes simplex virus infections. Pediatrics 2001;108:230-8.

79 Kimberlin DW, Lin CY, Jacobs RF, Powell DA, Frenkel LM, Gruber WC, et al. Natural history of neonatal herpes simplex virus infections in the acyclovir era. Pediatrics 2001;108:223-9.

\section{Appendix}

Die Schweizer Empfehlungen für das Management des Herpes genitalis und der Herpes-simplex-Virus-Infektion des Neugeborenen wurden von den folgenden Schweizer Fachgesellschaften gutgeheissen:

- Schweizerische Gesellschaft für Dermatologie und Venerologie;

- Schweizerische Gesellschaft für Urologie;

- Schweizerische Gesellschaft für Allergologie und Immunologie;

- Schweizerische Gesellschaft für Gynäkologie und Geburtshilfe;

- Schweizerische Gesellschaft für Ophthalmologie;

- Schweizerische Gesellschaft für Pädiatrie;

- Schweizerische Gesellschaft für Neonatologie;

- Schweizerische Gesellschaft für Infektiologie;

- Schweizerische Gesellschaft für Hämatologie;

- Schweizerische Gesellschaft für Onkologie;

- Schweizerische Gesellschaft für Innere Medizin;

- Schweizerische Gesellschaft für Allgemeinmedizin;

- Schweizerische Gesellschaft für Mikrobiologie. 